\title{
Conditional islet hypovascularisation does not preclude beta cell expansion during pregnancy in mice
}

\author{
Willem Staels ${ }^{1,2}$ - Yves Heremans ${ }^{1}$ - Gunter Leuckx ${ }^{1}$. Naomi Van Gassen ${ }^{1}$. \\ Ciro Salinno ${ }^{1}$ - Sofie De Groef ${ }^{1} \cdot$ Martine Cools $^{2}$ - Eli Keshet ${ }^{3} \cdot$ Yuval Dor $^{3}$ • \\ Harry Heimberg ${ }^{1}$ Nico De Leu ${ }^{1,4,5}$
}

Received: 30 September 2016 / Accepted: 23 February 2017 / Published online: 16 March 2017

(C) Springer-Verlag Berlin Heidelberg 2017

\begin{abstract}
Aims/hypothesis Endothelial-endocrine cell interactions and vascular endothelial growth factor (VEGF)-A signalling are deemed essential for maternal islet vascularisation, glucose control and beta cell expansion during mouse pregnancy. The aim of this study was to assess whether pregnancyassociated beta cell expansion was affected under conditions of islet hypovascularisation.

Methods Soluble fms-like tyrosine kinase 1 (sFLT1), a VEGF-A decoy receptor, was conditionally overexpressed in maternal mouse beta cells from 1.5 to 14.5 days post coitum. Islet vascularisation, glycaemic control, beta cell proliferation, individual beta cell size and total beta cell volume were assessed in both pregnant mice and non-pregnant littermates.
\end{abstract}

Electronic supplementary material The online version of this article (doi:10.1007/s00125-017-4243-1) contains peer-reviewed but unedited supplementary material, which is available to authorised users.

Harry Heimberg

Harry.Heimberg@vub.ac.be

$\triangle$ Nico De Leu

Nico.De.Leu@vub.ac.be

1 Beta Cell Neogenesis (BENE), Vrije Universiteit Brussel, Laarbeeklaan 103, 1090 Brussels, Belgium

2 Department of Paediatrics, Division of Paediatric Endocrinology, Ghent University Hospital and Ghent University, Ghent, Belgium

3 Department of Developmental Biology and Cancer Research, Institute of Medical Research Israel-Canada, Hebrew University Hadassah Medical School, Jerusalem, Israel

4 Department of Endocrinology, Universitair Ziekenhuis Brussel, Brussels, Belgium

5 Department of Endocrinology, Algemeen Stedelijk Ziekenhuis Aalst, Aalst, Belgium
Results Conditional overexpression of sFLT1 in beta cells resulted in islet hypovascularisation and glucose intolerance in both pregnant and non-pregnant mice. In contrast to nonpregnant littermates, glucose intolerance in pregnant mice was transient. sFLT1 overexpression did not affect pregnancy-associated changes in beta cell proliferation, individual beta cell size or total beta cell volume.

Conclusions/interpretation Reduced intra-islet VEGF-A signalling results in maternal islet hypovascularisation and impaired glycaemic control but does not preclude beta cell expansion during mouse pregnancy.

Keywords Beta cell · FLT1 · Islet · Pancreas · Pregnancy · Vascularisation · VEGF

$\begin{array}{ll}\text { Abbreviations } \\ \text { Dox } & \text { Doxycycline } \\ \text { dTg } & \text { Double transgenic } \\ \text { dTg NP } & \text { Double transgenic non-pregnant mice } \\ \text { dTg P } & \text { Double transgenic pregnant mice } \\ \text { dpc } & \text { Days post coitum } \\ \text { G } & \text { Gestational day } \\ \text { hGH } & \text { Human growth hormone } \\ \text { RIP } & \text { Rat insulin promoter } \\ \text { rtTA } & \text { Reverse tetracycline-dependent transactivator } \\ \text { sFLT1 } & \text { Soluble fms-like tyrosine kinase 1 } \\ \text { sTg } & \text { Single transgenic } \\ \text { VEGF } & \text { Vascular endothelial growth factor }\end{array}$

\section{Introduction}

Vascular endothelial growth factor (VEGF)-A signalling is crucial for normal pancreas development $[1,2]$, islet 
vascularisation [3] and glucose regulation [3, 4]. In contrast, interference with VEGF-A signalling and the resulting intraislet hypovascularisation do not hamper normal, agedependent augmentation of the adult beta cell mass or injuryinduced beta cell generation [5]. The question remains, however, whether VEGF-A and intra-islet endothelial cells are important for pregnancy-associated beta cell expansion and maternal glucose homeostasis. To address this issue, we used transgenic mice [5] in which beta cells conditionally produce soluble fmslike tyrosine kinase 1 (sFLT1) that acts as a VEGF-A decoy receptor. Overexpression of sFLT1 was induced from conception, blood glucose was monitored and, at gestational day (G)14.5, when maternal beta cell proliferation is maximal [6], islet vascularisation and beta cell adaptation were assessed.

\section{Methods}

Animal procedures Rat insulin promoter (RIP)-reverse tetracycline-dependent transactivator (rtTA) mice (RIPrtTA mice) and tetracycline operator (tetO)-sFLT1 mice (both on a mixed background, mainly CD1[ICR]) have been described previously $[5,7]$. Briefly, RIPrtTA mice were crossed with tetO-sFLT1 mice. In the resulting double transgenic (dTg) mice, sFLT1, a splice variant of the VEGF receptor 1, known to inhibit signalling by VEGF-A, VEGF-B and placental growth factor (reviewed in [8]), is expressed and secreted from beta cells when doxycycline (Dox) is administered via the drinking water. sFLT1 functions as a VEGF decoy receptor and reduces intra-islet VEGF-A bioavailability. All animal procedures were conducted ethically and in strict accordance with the Guide for the Care and Use of Laboratory Animals, eighth edition (2011), as well as with specific national laws and institutional guidelines. Mice were housed under standardised conditions $(12 \mathrm{~h}$ dark/12 h light cycle) and fed a standard diet ad libitum.

Genotyping was performed by PCR with gene-specific primers (see electronic supplementary material [ESM] Table 1). Genomic tail DNA of the transgenic strains was shown not to contain the human growth hormone $(\mathrm{hGH})$ mini-gene (see ESM Fig. 1), analysed as described in Oropeza et al [9]. Since the hGH
Fig. 1 Experimental design and validation of the mouse model in pregnancy. (a) Schematic representation of the RIPrtTA-tetO-sFLT1 transgenic mouse model and (b) the experimental design. (c, d) At G14.5, overt islet

hypovascularisation was seen in (c) dTg P+Dox mice (COLL-IV [red], insulin [green], DNA [blue]), compared with (d) dTg P -Dox mice, resulting in significant decreases in (e) the ratio of islet vessel area to total islet area and (f) islet vessel density. Data are shown as mean \pm SEM, $n=3-4$. Scale bar, $50 \mu \mathrm{m}$. White squares, dTg P -Dox; black squares, dTg P + Dox. ${ }^{*} p<0.05$ and ${ }^{* * *} p<0.001$, Student's $t$ test a

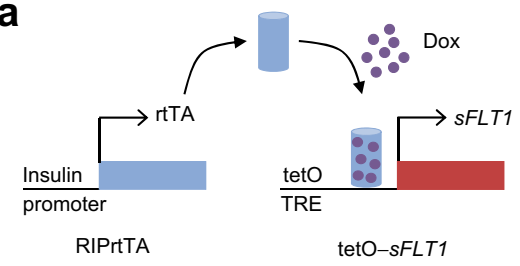

C
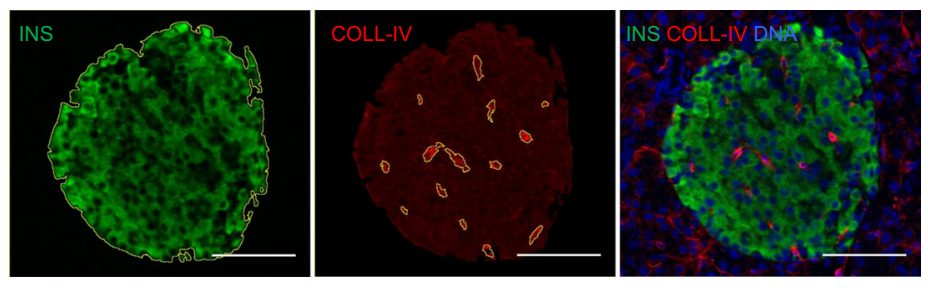

d
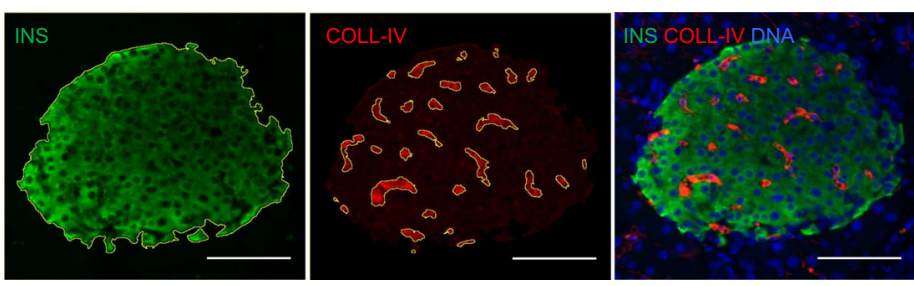

。

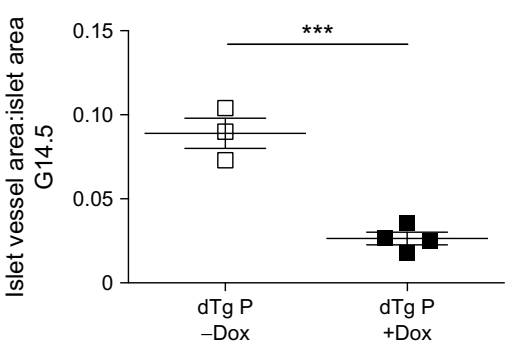

f

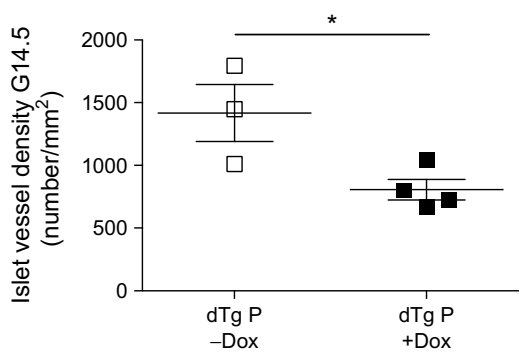



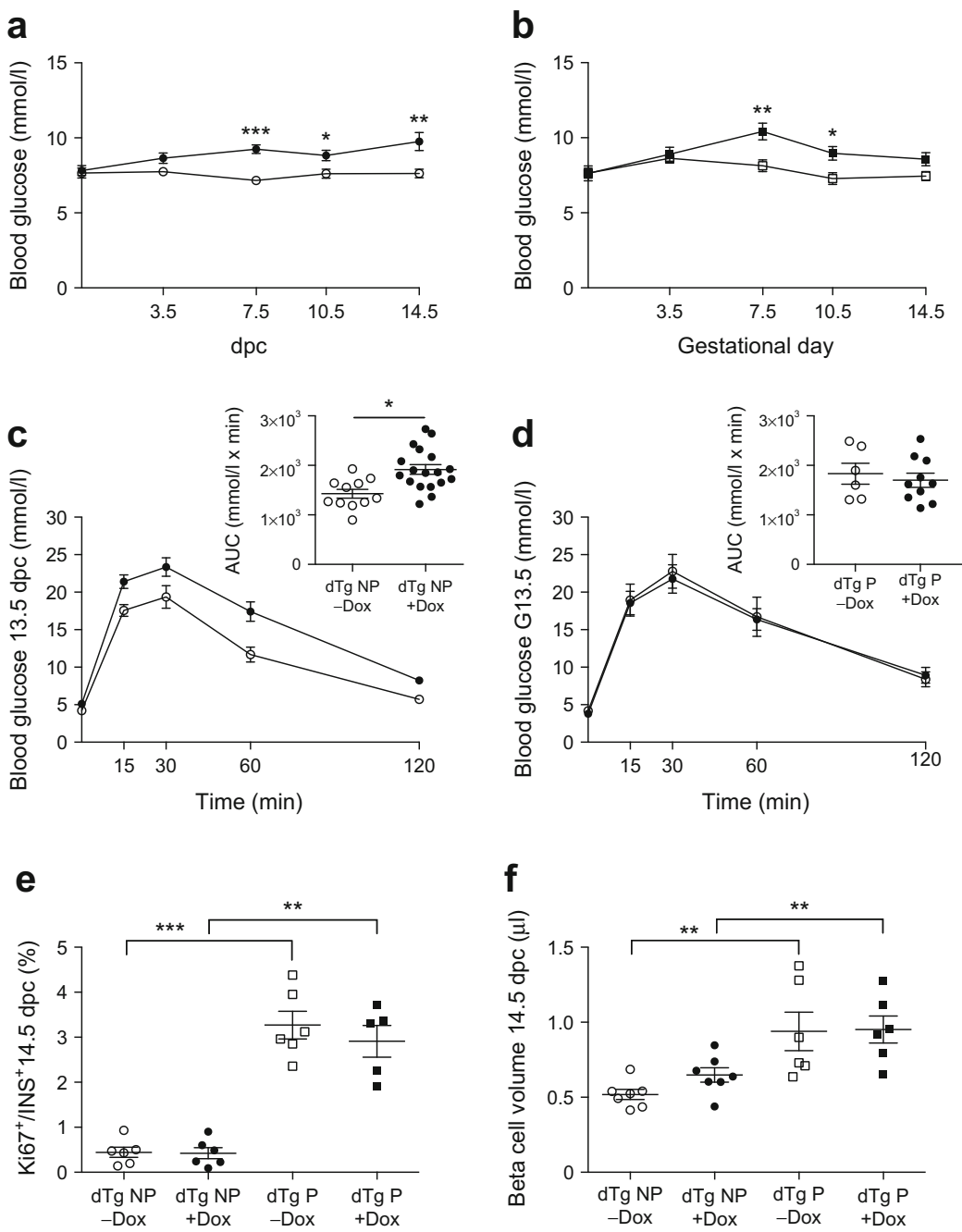

Fig. 2 The effect of islet hypovascularisation on metabolic control, beta cell proliferation rate and beta cell volume. (a) In non-pregnant mice, islet hypovascularisation resulted in increased $2 \mathrm{~h}$ fasting blood glucose from $7.5 \mathrm{dpc}$ onwards. (b) During pregnancy, islet hypovascularisation resulted in a transiently increased fasting glucose that normalised by G14.5. Data are shown as mean \pm SEM $(n=6-18)$. (c) In non-pregnant mice, islet hypovascularisation resulted in impaired glucose clearance at $13.5 \mathrm{dpc}$. (d) In pregnant mice, glucose clearance at G13.5 was similar in the dTg P -Dox and dTg P+Dox groups. The insets show the AUCs for each IPGTT, with lines indicating the mean \pm SEM $(n=6-18)$. (e) The percentage of $\mathrm{Ki}^{+} 7^{+}$insulin ${ }^{+}\left(\mathrm{INS}^{+}\right.$) cells was markedly increased at G14.5 in dTg P compared with dTg NP mice. Beta cell proliferation was not

significantly affected by Dox administration per se, nor was a significant interaction observed between (non-)pregnancy (NP vs P) and Dox administration (-Dox vs +Dox) (see also ESM Fig. 4). Data are shown as mean \pm SEM $(n=5-6)$. (f) Total beta cell volume was also increased at G14.5 in pregnant mice compared with non-pregnant littermates. Beta cell volume was not significantly affected by Dox administration per se, nor was a significant interaction observed between (non-)pregnancy and Dox administration (see also ESM Fig. 4). Data are shown as mean $\pm \operatorname{SEM}(n=6-7)$. Circles, dTg NP; squares, dTg P; white symbols, -Dox; black symbols, +Dox. ${ }^{*} p<0.05,{ }^{* *} p<0.01$; and ${ }^{* * *} p<0.001$, (a, b) Student's $t$ test and $(\mathbf{c}-\mathbf{f})$ two-way ANOVA with Tukey post hoc comparison

mini-gene acts as a lactogen on beta cells, inducing pregnancyrelated changes [10], its absence was mandatory for the study.

Nulliparous female RIPrtTA-tetO-sFLT1 mice were set up for timed mating with Crl:CD1(ICR) male mice (Janvier, Saint-Berthevin, France) at 8 weeks of age. Dox $(0.4 \mathrm{mg} / \mathrm{ml}$, Sigma-Aldrich, St Louis, MO, USA) was administered via drinking water (protected against light, $\mathrm{pH} 3.0,2 \%$ [wt/vol.] sucrose) from 1.5 until 14.5 days post coitum (dpc), henceforth referred to as gestational day $(\mathrm{G})$ in pregnant mice. Glucose tolerance was evaluated by IPGTT before timed mating and at $13.5 \mathrm{dpc}$ (see ESM Methods for further details). The

transgenic mouse model and the experimental design are schematically represented in Fig. 1a, b.

Protein analysis Pancreas samples were fixed in 10\% (vol./ vol.) neutral-buffered formalin before paraffin embedding. For primary antibodies, see ESM Table 2 . Secondary antibodies were labelled with cyanine or AlexaFluor (Jackson ImmunoResearch, Newmarket, UK). Hoechst 33342 (Sigma-Aldrich) was used for nuclear counterstaining. Sections were imaged using an Olympus BX61 (Tokyo, Japan) or, for whole-section analyses, a Nikon TE2000-E 
(Tokyo, Japan) microscope equipped with a Marzhauser Tango stage (Wetzlar, Germany). Pictures were analysed using Fiji image processing software [11]. Quantitation of beta cell proliferation was assessed by Ki67 immunoreactivity. Morphometric analysis of beta cell volume was done on at least $3 \%$ of the pancreas, as previously described [12]. Mean beta cell size was determined from the mean cross-sectional area per beta cell. See ESM Methods for further details.

Measurement of vascularisation Blood vessels and their basement membrane were visualised using an anti-collagen IV (COLL-IV) antibody. The ratios of intra-islet vessel area to insulin area and the intra-islet vessel densities (vessels $/ \mathrm{mm}^{2}$ of insulin-positive area) were analysed by Fiji image processing software as previously described [5]. Additional validation included immunostaining for CD31 as an endothelial cell marker and i.v. injected biotinylated lectin as a marker for functional blood vessels (Lycopersicon esculentum; Vector Laboratories, Burlingame, CA, USA; see ESM Fig. 2e-h), while hypoxia was detected with the oxygenation marker pimonidazole $(60 \mathrm{mg} / \mathrm{kg}$ body weight i.v.; HPI, Burlington, MA, USA), as described previously [5] (see ESM Fig. 2i-l).

Statistical analyses Two-tailed unpaired Student's $t$ tests or two-way ANOVA were used for data analysis as indicated (GraphPad Prism v7.0, San Diego, CA, USA). Data are presented as mean \pm SEM. Univariate scatterplots were used as much as possible to convey the full distribution of the data. A probability value of $p<0.05$ was considered statistically significant.

\section{Results}

Dox-administered dTg pregnant mice (dTg P) expressed the VEGF decoy receptor sFLT1 in $80 \%$ of their beta cells (see ESM Fig. 2a-d), and showed an overt regression of intra-islet endothelial cells and their supporting basement membrane at G14.5 (Fig. 1c, d and ESM Fig. 2e-h). The ratio of islet vessel area:total islet area decreased by $70 \%(0.089 \pm 0.009$ in dTg P in the absence of Dox (-Dox) vs $0.027 \pm 0.004$ in dTg P+Dox; Fig. 1e). Islet vessel density decreased by $43 \%$ compared with dTg P-Dox $\left(1418 \pm 227\right.$ vessels $/ \mathrm{mm}^{2}$ in dTg P-Dox vs $808 \pm 82$ vessels $/ \mathrm{mm}^{2}$ in dTg P+Dox; Fig. 1f), and by $56 \%$ and $57 \%$, respectively, compared with single transgenic (sTg) $\mathrm{P}+$ Dox (data not shown). Pimonidazole precipitation in $\mathrm{dTg}$ P+Dox indicated intra-islet hypoxia (see ESM Fig. 2i-1). The effect of islet hypovascularisation and hypoxia on glucose homeostasis and glucose tolerance was assessed by comparing $2 \mathrm{~h}$ fasting blood glucose and IPGTT values before and at the end of Dox administration (Fig. 2a, b shows blood glucose during the study period, while IPGTT results are shown in ESM Fig. 3a, b [before Dox] and Fig. 2c, d [end of Dox]).
Non-pregnant littermates were included to discriminate pregnancy-induced changes in glucose metabolism from islet hypovascularisation-induced changes. In dTg non-pregnant littermates (dTg NP), Dox administration resulted in impaired fasting glucose and glucose intolerance compared with $\mathrm{dTg}$ NP-Dox (Fig. 2a, c). In dTg P+Dox, fasting blood glucose was transiently increased at G7.5 and G10.5 but was comparable with dTg P-Dox at G14.5 (Fig. 2b), in line with the IPGTT at G13.5 (Fig. 2d). Glucose tolerance in dTg P+Dox at G0 and G13.5 was comparable with dTg P-Dox and sTg $\mathrm{P}+$ Dox (data not shown). The increase in body weight from G0 to G14.5 was similar in dTg P-Dox, dTg P+Dox (see ESM Fig. 3c) and sTg P+Dox (data not shown).

Beta cell proliferation, as determined by Ki67 immunostaining, increased 7-8-fold in pregnant mice at G14.5 compared with non-pregnant littermates. Pregnancy-associated hyperproliferation of beta cells in $\mathrm{dTg}$ mice was independent of Dox administration $\left(0.44 \pm 0.11 \% \mathrm{Ki}^{6} 7^{+}\right.$insulin $^{+}$cells in dTg NP-Dox vs $0.42 \pm 0.30 \%$ in $\mathrm{dTg}$ NP+Dox vs $3.27 \pm 0.31 \%$ in $\mathrm{dTg} \mathrm{P}-$ Dox vs $2.91 \pm 0.35 \%$ in $\mathrm{dTg} \mathrm{P}+$ Dox; Fig. 2e) and comparable with that in sTg littermates (see ESM Fig. 3d). Similarly, total beta cell volume was increased 1.5-2-fold in pregnant mice at G14.5 compared with non-pregnant littermates, independent of Dox administration $(0.52 \pm 0.03 \mu \mathrm{l}$ in $\mathrm{dTg} \mathrm{NP}-$ Dox vs $0.65 \pm 0.05 \mu \mathrm{l}$ in $\mathrm{dTg}$ $\mathrm{NP}+$ Dox vs $0.94 \pm 0.13$ in $\mathrm{dTg} \mathrm{P}-$ Dox vs $0.95 \pm 0.09 \mu \mathrm{l}$ in dTg P+Dox; Fig. 2f). Furthermore, at G14.5, individual beta cell size was increased in pregnant mice compared with nonpregnant littermates, again irrespective of Dox $\left(160 \pm 5 \mu \mathrm{m}^{2}\right.$ in dTg NP-Dox vs $170 \pm 8 \mu^{2}$ in $\mathrm{dTg} \mathrm{NP}+$ Dox vs $184 \pm 5 \mu \mathrm{m}^{2}$ in dTg P-Dox vs $190 \pm 9 \mu^{2}$ in dTg P+Dox; ESM Fig. 3e).

The summary data from the two-way ANOVA of AUC IPGTT at $13.5 \mathrm{dpc}$, beta cell proliferation, total beta cell volume and individual beta cell size are additionally shown in ESM Fig. 4 and described in ESM Table 3.

\section{Discussion}

VEGF-A is a master regulator of endothelial-beta cell crosstalk during beta cell development and regeneration [1, $13,14]$. By using a previously described transgenic mouse model that allows conditional beta cell-specific overexpression of sFLT1 [5], we investigated the effect of reduced intra-islet VEGF-A bioavailability on islet vascularisation, blood glucose control and beta cell expansion in pregnant mice. Concordant with its previously reported effect in nonpregnant mice [5], overexpression of sFLT1 resulted in a clear regression of islet endothelial cells and their supporting basement membrane. This effect was associated with overt intraislet hypoxia and glucose intolerance at mid-gestation. The latter is in line with previous reports that demonstrated 
impaired insulin secretion on intra-islet hypovascularisation or hypoxia in vivo $[5,15,16]$. Indeed, preliminary data indicated impaired glucose-stimulated insulin secretion in vivo. However, isolated hypovascularised islets were not functionally impaired. Such an apparent in vivo/in vitro discrepancy has been reported previously and suggests that glucose intolerance results from a delay in glucose sensing or insulin diffusion rather than beta cell dysfunction per se $[5,16]$. Interestingly, and as opposed to non-pregnant littermates, glucose intolerance was only transient as blood glucose spontaneously normalised in pregnant mice between G10.5 and G14.5. It is likely that glucose intolerance seen at G7.5 was mitigated by beta cell-adaptive changes that occur beyond this time point. Pregnant mice overexpressing sFLT1 indeed showed all previously reported beta cell adaptations [17], i.e. both hyperplasia and hypertrophy, resulting in a significant increase in total beta cell volume at G14.5. Our data thus provide in vivo evidence that weakens the presumed importance of the endothelial-endocrine axis in pregnancy-associated beta cell expansion. This appears to contradict a previous report [18] in which islet endothelial cell proliferation preceded beta cell proliferation in pregnant rats, and purified islet-derived proliferating endothelial cells stimulated beta cell proliferation in vitro. While these data indirectly suggest that endothelial cellderived signals promote beta cell proliferation in the context of pregnancy, our current in vivo data tone down the actual importance of endothelial cells for pregnancyassociated beta cell proliferation. Whether this difference in outcome is related to study design (i.e. in vitro vs in vivo) or species is presently not clear. In mice with islet hypovascularisation, it cannot unequivocally be excluded that the residual islet vasculature still contributes to the observed beta cell adaptations. Moreover, our current data cannot exclude that cell cycle-activating mechanisms under hypoxic conditions (e.g. mechanistic target of rapamycin complex 1 [mTORC1] signalling) may mask differences in beta cell proliferation.

Taken together, the results obtained with conditional reduction of VEGF-A signalling in islets of pregnant mice support the importance of this signalling for vessel maintenance and tight glucose control until mid-gestation but, in contrast to interference with prolactin [19] or serotonin [20] signalling, it does not preclude pregnancy-associated beta cell expansion.

Acknowledgements The authors wish to thank V. Laurysens, A. Demarré, E. Quartier and G. Stangé (Diabetes Research Center, Vrije Universiteit Brussel) for technical assistance and J. Estall (Montreal Diabetes Research Center) for providing control gDNA samples for human growth hormone (hGH) PCR and sharing expertise. The authors are also grateful for the support of Universitaire Stichting van België.
Data availability The datasets generated and/or analysed during the current study are available from the corresponding author on reasonable request.

Funding The authors acknowledge support by grants from the Institute for the Promotion of Innovation by Science and Technology in Flanders (IWT), the Research Foundation-Flanders (FWO), the VUB Research Council, Stichting Diabetes Onderzoek Nederland, the European Union Sixth and Seventh Framework Programmes, the Wetenschappelijk Fonds Willy Gepts (WFWG) of the UZ Brussel and the Belgian Federal Science Policy (IAPVII-07).

Duality of interest The authors declare that there is no duality of interest associated with this manuscript.

Contribution statement The study was conceived and designed by WS, NVG, HH and NDL. Data acquisition was performed by WS, YH, GL, NVG, SDG and CS. Data analysis and interpretation was done by WS, EK, YD, MC, HH and NDL. WS, YH, YD, HH and NDL drafted the manuscript, EK, YD, MC and SDG contributed to the discussion. All authors revised the manuscript and approved the final version to be published. NDL is the guarantor of this work.

\section{References}

1. Lammert E, Cleaver O, Melton D (2001) Induction of pancreatic differentiation by signals from blood vessels. Science 294:564-567

2. Yoshitomi H, Zaret KS (2004) Endothelial cell interactions initiate dorsal pancreas development by selectively inducing the transcription factor Ptfla. Development 131:807-817

3. Lammert E, Gu G, McLaughlin M et al (2003) Role of VEGF-A in vascularization of pancreatic islets. Curr Biol 13:1070-1074

4. Nikolova G, Jabs N, Konstantinova I et al (2006) The vascular basement membrane: a niche for insulin gene expression and $\beta$ cell proliferation. Dev Cell 10:397-405

5. D'Hoker J, De Leu N, Heremans Y et al (2013) Conditional hypovascularization and hypoxia in islets do not overtly influence adult beta-cell mass or function. Diabetes 62:4165-4173

6. Parsons JA, Brelje TC, Sorenson RL (1992) Adaptation of islets of Langerhans to pregnancy: increased islet cell proliferation and insulin secretion correlates with the onset of placental lactogen secretion. Endocrinology 130:1459-1466

7. May D, Gilon D, Djonov V et al (2008) Transgenic system for conditional induction and rescue of chronic myocardial hibernation provides insights into genomic programs of hibernation. Proc Natl Acad Sci U S A 105:282-287

8. Shibuya M (2006) Vascular endothelial growth factor receptor-1 (VEGFR-1/Flt-1): a dual regulator for angiogenesis. Angiogenesis 9:225-230

9. Oropeza D, Jouvet N, Budry L et al (2015) Phenotypic characterization of MIP-CreERT1Lphi mice with transgene-driven islet expression of human growth hormone. Diabetes 64:3798-3807

10. Brouwers B, de Faudeur G, Osipovich AB et al (2014) Impaired islet function in commonly used transgenic mouse lines due to human growth hormone minigene expression. Cell Metab 20: 979-990

11. Schindelin J, Arganda-Carreras I, Frise E et al (2012) Fiji: an opensource platform for biological-image analysis. Nat Methods 9:676682

12. Coppens V, Heremans Y, Leuckx G et al (2013) Human blood outgrowth endothelial cells improve islet survival and function when co-transplanted in a mouse model of diabetes. Diabetologia $56: 382-390$ 
13. Cleaver O, Dor Y (2012) Vascular instruction of pancreas development. Development 139:2833-2843

14. Brissova M, Aamodt K, Brahmachary P et al (2014) Islet microenvironment, modulated by vascular endothelial growth factor-a signaling, promotes beta cell regeneration. Cell Metab 19:498-511

15. Cantley J, Grey ST, Maxwell PH, Withers DJ (2010) The hypoxia response pathway and $\beta$-cell function. Diabetes Obes Metab 12: $159-167$

16. Kragl M, Schubert R, Karsjens H et al (2016) The biomechanical properties of an epithelial tissue determine the location of its vasculature. Nat Commun 7:13560
17. Rieck S, Kaestner KH (2010) Expansion of beta-cell mass in response to pregnancy. Trends Endocrinol Metab 21:151-158

18. Johansson M, Mattsson G, Andersson A, Jansson L, Carlsson PO (2006) Islet endothelial cells and pancreatic beta-cell proliferation: studies in vitro and during pregnancy in adult rats. Endocrinology 147:2315-2324

19. Banerjee RR, Cyphert HA, Walker EM et al (2016) Gestational diabetes mellitus from inactivation of prolactin receptor and MafB in islet beta-cells. Diabetes 65:2331-2341

20. Kim H, Toyofuku Y, Lynn FC et al (2010) Serotonin regulates pancreatic beta cell mass during pregnancy. Nat Med 16:804-808 